С. В. Марвин. Начально-краевая задача электродинамики для дефектного ферритового тела

УДК 517.968 .73

DOI: $10.18101 / 2304-5728-2019-1-31-40$

\title{
НАЧАЛЬНО-КРАЕВАЯ ЗАДАЧА ЭЛЕКТРОДИНАМИКИ ДЛЯ ДЕФЕКТНОГО ФЕРРИТОВОГО ТЕЛА
}

\section{(C) Марвин Сергей Владимирович}

кандидат физико-математических наук, докторант, доцент, Уральский федеральный университет имени первого

Президента России Б. Н. Ельцина

Россия, 620002, г. Екатеринбург, ул. Мира, 19

E-mail: s.v.marvin@yandex.ru

Рассмотрена начально-краевая задача для уравнений Максвелла применительно к ферритовому телу, имеющему структурные дефекты. Для постановки начально-краевой задачи выбран функциональный класс, учитывающий условия сопряжения на границе раздела двух сред, не являющихся идеальными проводниками. Векторные поля выбранного функционального класса квадратично суммируемы во всем пространстве и имеют квадратично суммируемые обобщенные роторы. Кроме того, векторные поля из выбранного функционального класса дифференцируемы по времени в смысле сходимости по среднеквадратичной норме. При широких предположениях, касающихся зависимости электропроводности, диэлектрической и магнитной проницаемости феррита от пространственных координат, и при естественных допущениях, касающихся характера зависимости стороннего тока от времени, показано, что в выбранном функциональном классе существует единственное решение рассмотренной начально-краевой задачи и это решение непрерывно зависит от начальных условий.

Ключевые слова: уравнения Максвелла; начальные условия; условия сопряжения; среднеквадратичная норма; банахово пространство; теорема существования; обобщенный ротор; интегро-дифференциальные уравнения; замкнутый оператор; обратный оператор.

Для цитирования:

Марвин C. В. Начально-краевая задача электродинамики для дефектного ферритового тела // Вестник Бурятского государственного университета. Математика, информатика. 2019. № 1. С. 31-40.

\section{Введение}

Начально-краевые задачи электродинамики, не предполагающие сокращающуюся гармоническую зависимость электромагнитного поля от времени, необходимы как для общего описания, так и для конкретных расчетов нестационарных электромагнитных полей. Нестационарные электромагнитные поля возникают в электротехнических установках при переходных процессах; также они используются в неразрушающем контроле проводящих изделий [1]. С этим обстоятельством связана актуальность исследования начально-краевых задач электродинамики, в том числе в проводящих магнитных средах. В частности, необходимо доказывать 
существование и единственность решения таких начально-краевых задач, это служит базой для численных методов.

Ранее широко исследовались внутренние начально-краевые задачи электродинамики для ограниченных областей $[2 ; 3]$; наиболее существенные результаты были получены для областей с границами класса $C^{(2)}$ [3]. Однако в электротехнике и особенно в неразрушающем контроле актуальны не только внутренние начально-краевые задачи, но также и задачи сопряжения, в постановке которых граничные условия связывают поле снаружи проводящего тела с полем внутри тела: именно эта связь (безусловно, физически реальная) делает неразрушающий контроль возможным, позволяет судить о внутренней структуре проводника по внешнему полю.

Начально-краевые задачи сопряжения для магнитных сред были рассмотрены ранее применительно к дефектному ферромагнитному металлу [4] и магнитному диэлектрику, имеющему структурные нарушения [5]. Однако в электротехнических устройствах используются также ферриты, представляющие собой магнитные полупроводники. Ферритовые сердечники в трансформаторах, дросселях и иных стабилизирующих устройствах регулярно подвергаются воздействию нестационарных электромагнитных полей при случайных изменениях силы тока в обмотках; кроме того, феррит может быть целенаправленно подвергнут воздействию нестационарного электромагнитного поля для выявления его структурных нарушений [1].

Таким образом, исследования начально-краевых задач сопряжения применительно к ферритовым телам актуальны; в частности, актуально доказательство существования и единственности решения этих задач.

\section{1 Постановка задачи}

Для множества вещественных чисел примем стандартное обозначение $\mathrm{R}$. Соответственно, трехмерное геометрическое пространство отождествим с декартовой степенью $\mathrm{R}^{3}$. Упорядоченный набор трех пространственных координат точки будем обозначать $\boldsymbol{r}$.

Предположим, что феррит занимает ограниченную область $\Omega \subset \mathrm{R}^{3}$; граница области $\Omega$ представляет собой кусочно-гладкую поверхность. Дефектные области $\Omega_{1}, \Omega_{2}, \ldots, \Omega_{k}$ имеют кусочно-гладкие границы; замыкания этих областей включаются в $\Omega$ и не пересекаются: $\bar{\Omega}_{i} \subset \Omega$, $\bar{\Omega}_{i} \cap \bar{\Omega}_{j}=\varnothing$ при $i \neq j$.

Ферриты представляют собой магнетики с магнитной проницаемостью $\mu$, существенно превосходящей 1 . В то же время ферриты являются полупроводниками. То есть в этих материалах наличествуют как свободные электроны, благодаря которым феррит обладает ненулевой электропроводностью $\sigma$, так и связанные электроны, обеспечивающие во внешнем 
C. В. Марвин. Начально-краевая задача электродинамики для дефектного ферритового тела

электрическом поле поляризацию достаточно сильную, чтобы диэлектрическая проницаемость ферритов $\varepsilon$ существенно превышала 1 .

К дефектам приводят технологические недочеты изготовления ферритов: неравномерное перемешивание шихты - смеси порошков исходных оксидов, из которых изготовлен феррит; последующее некачественное прессование и спекание порошков. В результате этих недочетов и возникают дефектные области, на границе которых электрические и магнитные свойства ферритов резко, скачкообразно изменяются. Это проявляется в координатной зависимости электропроводности, диэлектрической и магнитной проницаемости феррита.

В рамках рассматриваемой задачи примем естественное предположение, что электропроводность, диэлектрическая и магнитная проницаемость феррита не зависят от времени. Как функция пространственных координат $\sigma(\boldsymbol{r})>0$, непрерывна в областях $\Omega_{i}$ и $\Omega \backslash\left(\bigcup_{i=1}^{k} \bar{\Omega}_{i}\right)$, причем может быть непрерывно продолжена с каждой из этих областей на ее границу, оставаясь при этом положительной. Кроме того, $\varepsilon(\boldsymbol{r})>1, \mu(\boldsymbol{r})>1$, причем функции $\varepsilon(\boldsymbol{r})$ и $\mu(\boldsymbol{r})$ непрерывны в $\Omega_{i}$ и $\Omega \backslash\left(\bigcup_{i=1}^{k} \bar{\Omega}_{i}\right)$ и также допускают непрерывное продолжение с каждой из этих областей на границу, оставаясь при таком продолжении больше 1. При переходе же через границы $\Omega_{i}$ функции $\sigma(\boldsymbol{r}), \varepsilon(\boldsymbol{r})$ и $\mu(\boldsymbol{r})$ терпят разрыв. Снаружи ферритового тела, то есть в точках, внешних по отношению к $\Omega, \sigma(\boldsymbol{r}) \equiv 0$ и $\varepsilon(\boldsymbol{r}) \equiv \mu(\boldsymbol{r}) \equiv 1$.

Будем предполагать, что сторонний ток сосредоточен в ограниченной области $T$; замыкания областей $\Omega$ и $T$ не пересекаются: $\bar{\Omega} \cap \bar{T}=\varnothing$. Как функция пространственных координат $\boldsymbol{r}$ и времени $t$, плотность стороннего тока $\boldsymbol{J}(\boldsymbol{r}, t)$ дважды непрерывно дифференцируема при $\boldsymbol{r} \in \bar{T}$ и $t \geq 0$; кроме того, $\boldsymbol{J}(\boldsymbol{r}, 0) \equiv \boldsymbol{O}$ ( $\boldsymbol{0}$ - обозначение нулевого вектора). В точках, внешних по отношению к $T, \boldsymbol{J}(\boldsymbol{r}, t) \equiv \boldsymbol{0}$.

Электромагнитное поле внутри и снаружи ферритового тела удовлетворяет системе уравнений Максвелла:

$$
\left\{\begin{array}{l}
\frac{\partial \boldsymbol{E}}{\partial t}=\frac{1}{\varepsilon_{0} \varepsilon(\boldsymbol{r})} \operatorname{rot} \boldsymbol{H}-\frac{\sigma(\boldsymbol{r})}{\varepsilon_{0} \varepsilon(\boldsymbol{r})} \boldsymbol{E}-\frac{1}{\varepsilon_{0} \varepsilon(\boldsymbol{r})} \boldsymbol{J}(\boldsymbol{r}, t) \\
\frac{\partial \boldsymbol{H}}{\partial t}=-\frac{1}{\mu_{0} \mu(\boldsymbol{r})} \operatorname{rot} \boldsymbol{E}
\end{array},\right.
$$

где $\boldsymbol{E}$ и $\boldsymbol{H}$ - соответственно напряженности электрического и магнитного поля; $\varepsilon_{0}$ и $\mu_{0}-$ соответственно диэлектрическая и магнитная постоянные. 
В точках гладкости границ областей $\Omega_{i}$ и $\Omega$ электромагнитное поле удовлетворяет условиям сопряжения для двух сред, не являющихся идеальными проводниками:

$$
\left\{\begin{array}{l}
\boldsymbol{E}_{\tau, \mathrm{int}}=\boldsymbol{E}_{\tau, \mathrm{ext}} \\
\boldsymbol{H}_{\tau, \mathrm{int}}=\boldsymbol{H}_{\tau, \mathrm{ext}}
\end{array}\right.
$$

где индекс $\tau$ обозначает касательную компоненту вектора; int и ext обозначение предела соответственно изнутри и снаружи области.

Электромагнитное поле в начальный момент времени $t=0$ определяется начальными условиями:

$$
\left\{\begin{array}{l}
\boldsymbol{E}(\boldsymbol{r}, 0)=\boldsymbol{E}_{0}(\boldsymbol{r}) \\
\boldsymbol{H}(\boldsymbol{r}, 0)=\boldsymbol{H}_{0}(\boldsymbol{r})
\end{array} .\right.
$$

Начальное магнитное поле должно удовлетворять условию $\operatorname{div}\left(\mu(\boldsymbol{r}) \boldsymbol{H}_{0}(\boldsymbol{r})\right) \equiv 0$, где частные производные, входящие в дивергенцию, в общем случае следует понимать как обобщенные. Заметим, что в силу уравнений (1) выполнение этого тождества для магнитного поля при $t=0$ влечет за собой его выполнение при $t>0[4 ; 6]$. В остальном выбор $\boldsymbol{E}_{0}(\boldsymbol{r})$ и $\boldsymbol{H}_{0}(\boldsymbol{r})$ может быть произвольным в рамках функционального класса, определяемого для постановки задачи.

Для решения задачи (1)-(3) функциональный класс выберем следующим образом. В любой фиксированный момент времени $t \geq 0$ векторные поля $\boldsymbol{E}(\boldsymbol{r}, t)$ и $\boldsymbol{H}(\boldsymbol{r}, t)$ должны принадлежать пространству $\mathbf{H}\left(\operatorname{rot}, \mathrm{R}^{3}\right)-$ пространству векторных полей, квадратично суммируемых в $\mathrm{R}^{3}$ вместе со своими обобщенными роторами [3-5]. Кроме того, при $t \geq 0 \boldsymbol{E}(\boldsymbol{r}, t)$ и $\boldsymbol{H}(\boldsymbol{r}, t)$ должны быть дифференцируемы по времени в смысле сходимости по среднеквадратичной норме в $\mathrm{R}^{3}$ :

$$
\begin{gathered}
\left\|\frac{\boldsymbol{E}(\boldsymbol{r}, t+\Delta t)-\boldsymbol{E}(\boldsymbol{r}, t)}{\Delta t}-\boldsymbol{E}_{t}^{\prime}(\boldsymbol{r}, t)\right\|_{2} \underset{\Delta t \rightarrow 0}{\rightarrow} 0, \\
\left\|\frac{\boldsymbol{H}(\boldsymbol{r}, t+\Delta t)-\boldsymbol{H}(\boldsymbol{r}, t)}{\Delta t}-\boldsymbol{H}_{t}^{\prime}(\boldsymbol{r}, t)\right\|_{2}^{\rightarrow} \underset{\Delta t \rightarrow 0}{\rightarrow} 0,
\end{gathered}
$$

где индекс 2 означает среднеквадратичную норму на $\mathrm{R}^{3}$.

Покажем, что в такой постановке у начально-краевой задачи (1)-(3) существует единственное решение, непрерывно зависящее от начальных условий.

\section{2 Основные теоремы}

Для исследования начально-краевой задачи (1)-(3) проведем исследование свойств дифференциального оператора $\hat{A}$, который на каждую упорядоченную пару векторных полей $(\boldsymbol{u} ; \boldsymbol{v}) \in\left(\mathbf{H}\left(\operatorname{rot}, \mathrm{R}^{3}\right)\right)^{2}$ действует 
C. В. Марвин. Начально-краевая задача электродинамики для дефектного ферритового тела

следующим образом:

$$
\hat{A}(\boldsymbol{u} ; \boldsymbol{v})=\left(\frac{1}{\varepsilon_{0} \varepsilon} \operatorname{rot} v-\frac{\sigma}{\varepsilon_{0} \varepsilon} \boldsymbol{u} ;-\frac{1}{\mu_{0} \mu} \operatorname{rot} \boldsymbol{u}\right) .
$$

Для того чтобы сформулировать свойства пространства $\mathbf{H}\left(\operatorname{rot}, \mathrm{R}^{3}\right)$ и оператора $\hat{A}$, введем следующие обозначения. $\mathbf{H}^{\prime}-$ пространство непрерывно дифференцируемых в $\mathrm{R}^{3}$ векторных полей, квадратично суммируемых вместе со своими роторами. $\mathbf{K}$ - пространство векторных полей, непрерывно дифференцируемых в областях $\Omega_{i}, \Omega \backslash\left(\bigcup_{i=1}^{k} \bar{\Omega}_{i}\right)$ и $\mathrm{R}^{3} \backslash \bar{\Omega}$, допускающих непрерывное продолжение вместе со своими производными с каждой из этих областей на ее границу, удовлетворяющих граничным условиям вида (2) и, кроме того, квадратично суммируемых в $\mathrm{R}^{3}$ вместе со своими роторами. $\mathbf{L}_{2}-$ пространство векторных полей, квадратично суммируемых на множестве, указанном в скобках после $\mathbf{L}_{2}$. $\hat{A}^{\prime}$ - дифференциальный оператор, действующий, как и оператор $\hat{A}$, по формуле (4), но на меньшем пространстве $\mathbf{K}$.

Ранее была доказана серия теорем, касающихся $\mathbf{H}\left(\operatorname{rot}, \mathrm{R}^{3}\right)$ [4].

Tеорема 1. $\mathbf{H}^{\prime} \subset \mathbf{K} \subset \mathbf{H}\left(\operatorname{rot}, \mathrm{R}^{3}\right) \subset \mathbf{L}_{2}\left(\mathrm{R}^{3}\right)$, причем $\mathbf{H}^{\prime}-$ плотное подпространство $\mathbf{L}_{2}\left(\mathrm{R}^{3}\right)$.

Теорема 2. Если последовательности $\boldsymbol{u}_{n} \in \mathbf{H}\left(\operatorname{rot}, \mathrm{R}^{3}\right)$ и $\operatorname{rot} \boldsymbol{u}_{n}$ сходятся по норме $\mathbf{L}_{2}\left(\mathrm{R}^{3}\right)$, соответственно к $\boldsymbol{u}$ и $\boldsymbol{v}$, то $\boldsymbol{u} \in \mathbf{H}\left(\operatorname{rot}, \mathrm{R}^{3}\right)$ и $\boldsymbol{v}=\operatorname{rot} \boldsymbol{u}$.

Tеорема 3. Для любого векторного поля $\boldsymbol{u} \in \mathbf{H}\left(\operatorname{rot}, \mathrm{R}^{3}\right)$ существует последовательность $\boldsymbol{u}_{n} \in \mathbf{H}^{\prime}$, сходящаяся по норме $\mathbf{L}_{2}\left(\mathrm{R}^{3}\right)$ к $\boldsymbol{u}$, для которой $\operatorname{rot} \boldsymbol{u}_{n}$ сходится к $\operatorname{rot} \boldsymbol{u}$.

Воспользовавшись теоремами 1-3, можно доказать следующую теоремy.

Tеорема 4. Оператор $\hat{A}$ имеет плотную в $\left(\mathbf{L}_{2}\left(\mathrm{R}^{3}\right)\right)^{2}$ область определения и является минимальным замкнутым расширением оператора $\hat{A}^{\prime}$.

Ранее аналогичное свойство оператора $\hat{A}$ было доказано для случаев ферромагнетика [4] и магнитодиэлектрика [5]. В рассматриваемом случае дефектного ферритового тела имеет место иной характер зависимости коэффициентов в выражении (4) от пространственных координат, так как электрические и магнитные свойства предполагаются уже другими. Однако рассуждения, методы и приемы, с помощью которых доказывается теорема 4, совершенно аналогичны тем, которые были использованы ранее; поэтому теорему можно принять как уже доказанную. 
Теорема 4 обосновывает выбор пространства $\mathbf{H}\left(\operatorname{rot}, \mathrm{R}^{3}\right)$ для постановки исследуемой начально-краевой задачи: оператор $\hat{A}$ является минимальным замкнутым расширением оператора $\hat{A}^{\prime}$, в то время как $\hat{A}^{\prime}$ определен на пространстве $\mathbf{K}$, учитывающем граничные условия задачи. При этом основные теоремы, касающиеся существования и единственности решения задачи Коши в абстрактном нормированном пространстве, доказаны исключительно для замкнутых операторов [1].

Tеорема 5. Для любой упорядоченной пары $(\boldsymbol{f}, \boldsymbol{g}) \in\left(\mathbf{L}_{2}\left(\mathrm{R}^{3}\right)\right)^{2}$ и для любого $\quad p>0$ у уравнения $\hat{A}(\boldsymbol{u} ; \boldsymbol{v})-p \cdot(\boldsymbol{u} ; \boldsymbol{v})=(\boldsymbol{f} ; \boldsymbol{g})$ в пространстве $\left(\mathbf{H}\left(\operatorname{rot}, \mathrm{R}^{3}\right)\right)^{2}$ существует единственное решение. Это решение удовлетворяет неравенству

$$
\sqrt{\varepsilon_{0}\|\sqrt{\varepsilon} \boldsymbol{u}\|_{2}^{2}+\mu_{0}\|\sqrt{\mu} \boldsymbol{v}\|_{2}^{2}} \leq \frac{1}{p} \cdot \sqrt{\varepsilon_{0}\|\sqrt{\varepsilon} \boldsymbol{f}\|_{2}^{2}+\mu_{0}\|\sqrt{\mu} \boldsymbol{g}\|_{2}^{2}} .
$$

Доказательство. Рассматриваемое уравнение с оператором $\hat{A}$ запишем в эквивалентной форме:

$$
\left\{\begin{array}{l}
-\operatorname{rot} v+\sigma(\boldsymbol{r}) \boldsymbol{u}+\varepsilon_{0} p \varepsilon(\boldsymbol{r}) \boldsymbol{u}=-\varepsilon_{0} \varepsilon(\boldsymbol{r}) \boldsymbol{f}(\boldsymbol{r}) \\
\operatorname{rot} \boldsymbol{u}+\mu_{0} p \mu(\boldsymbol{r}) \boldsymbol{v}=-\mu_{0} \mu(\boldsymbol{r}) \boldsymbol{g}(\boldsymbol{r})
\end{array} .\right.
$$

Для полей $\boldsymbol{u}, \boldsymbol{v} \in \mathbf{H}\left(\operatorname{rot}, \mathrm{R}^{3}\right)$ справедливо следующее равенство [4]:

$$
\int_{\mathrm{R}^{3}}(\boldsymbol{v}(\boldsymbol{r}) \operatorname{rot} \boldsymbol{u}(\boldsymbol{r})-\boldsymbol{u}(\boldsymbol{r}) \operatorname{rot} \boldsymbol{v}(\boldsymbol{r})) d V=0 .
$$

Путем умножения первого и второго уравнения системы (6) соответственно на $\boldsymbol{u}$ и $\boldsymbol{v}$, последующего сложения уравнений и интегрирования по $\mathrm{R}^{3}$, в силу (7), получаем равенство

$$
\begin{aligned}
& \varepsilon_{0} p\|\sqrt{\varepsilon} \boldsymbol{u}\|_{2}^{2}+\mu_{0} p\|\sqrt{\mu} \boldsymbol{v}\|_{2}^{2}+\int_{\Omega} \sigma(\boldsymbol{r})|\boldsymbol{u}(\boldsymbol{r})|^{2} d V= \\
& =-\varepsilon_{0} \int_{\mathrm{R}^{3}} \varepsilon(\boldsymbol{r}) \boldsymbol{f}(\boldsymbol{r}) \boldsymbol{u}(\boldsymbol{r}) d V-\mu_{0} \int_{\mathbb{R}^{3}} \mu(\boldsymbol{r}) \boldsymbol{g}(\boldsymbol{r}) \boldsymbol{v}(\boldsymbol{r}) d V .
\end{aligned}
$$

В полученном равенстве учтено, что $\sigma(\boldsymbol{r}) \neq 0$ только в $\Omega$. Заметим, что левая часть полученного равенства неотрицательна. Следовательно, в силу неравенства Коши - Буняковского для интегралов и сумм,

$$
\begin{gathered}
\varepsilon_{0} p\|\sqrt{\varepsilon} \boldsymbol{u}\|_{2}^{2}+\mu_{0} p\|\sqrt{\mu} \boldsymbol{v}\|_{2}^{2}+\int_{\Omega} \sigma(\boldsymbol{r})|\boldsymbol{u}(\boldsymbol{r})|^{2} d V= \\
=\left|\varepsilon_{0} \int_{\mathrm{R}^{3}} \varepsilon(\boldsymbol{r}) \boldsymbol{f}(\boldsymbol{r}) \boldsymbol{u}(\boldsymbol{r}) d V+\mu_{0} \int_{\mathrm{R}^{3}} \mu(\boldsymbol{r}) \boldsymbol{g}(\boldsymbol{r}) \boldsymbol{v}(\boldsymbol{r}) d V\right| \leq \\
\leq \varepsilon_{0}\|\sqrt{\varepsilon} \boldsymbol{u}\|_{2}\|\sqrt{\varepsilon} \boldsymbol{f}\|_{2}+\mu_{0}\|\sqrt{\mu} \boldsymbol{v}\|_{2}\|\sqrt{\mu} \boldsymbol{g}\|_{2} \leq
\end{gathered}
$$


C. В. Марвин. Начально-краевая задача электродинамики для дефектного ферритового тела

$$
\leq \sqrt{\varepsilon_{0}\|\sqrt{\varepsilon} \boldsymbol{u}\|_{2}^{2}+\mu_{0}\|\sqrt{\mu} \boldsymbol{v}\|_{2}^{2}} \cdot \sqrt{\varepsilon_{0}\|\sqrt{\varepsilon} \boldsymbol{f}\|_{2}^{2}+\mu_{0}\|\sqrt{\mu} \boldsymbol{g}\|_{2}^{2}} .
$$

Так как $\sigma(\boldsymbol{r})>0$, удаление третьего слагаемого в исходном выражении полученное неравенство только усилит:

$$
\varepsilon_{0} p\|\sqrt{\varepsilon} \boldsymbol{u}\|_{2}^{2}+\mu_{0} p\|\sqrt{\mu} \boldsymbol{v}\|_{2}^{2} \leq \sqrt{\varepsilon_{0}\|\sqrt{\varepsilon} \boldsymbol{u}\|_{2}^{2}+\mu_{0}\|\sqrt{\mu} \boldsymbol{v}\|_{2}^{2}} \cdot \sqrt{\varepsilon_{0}\|\sqrt{\varepsilon} \boldsymbol{f}\|_{2}^{2}+\mu_{0}\|\sqrt{\mu} \boldsymbol{g}\|_{2}^{2}} .
$$

Из последнего неравенства непосредственно вытекает (5).

Заметим, что в силу доказанного неравенства (5) при нулевых $\boldsymbol{f}$ и $\boldsymbol{g}$ решение у системы (6) может быть только тривиальным. Следовательно, при любых $\boldsymbol{f}$ и $\boldsymbol{g}$ система (6) имеет не более одного решения.

Определим интегральный оператор $\hat{P}$ следующим образом [1]: для любого векторного поля $\boldsymbol{w} \in \mathbf{L}_{2}\left(\mathrm{R}^{3}\right) \hat{P}[\boldsymbol{w}]=\int_{\mathrm{R}^{3}} G\left(p, \boldsymbol{r}, \boldsymbol{r}^{\prime}\right) \boldsymbol{w}\left(\boldsymbol{r}^{\prime}\right) d V^{\prime}$, где $G\left(p, \boldsymbol{r}, \boldsymbol{r}^{\prime}\right)=\exp \left(-p \sqrt{\varepsilon_{0} \mu_{0}}\left|\boldsymbol{r}-\boldsymbol{r}^{\prime}\right|\right) / 4 \pi\left|\boldsymbol{r}-\boldsymbol{r}^{\prime}\right| . \quad$ Интегро-дифференциальные операторы $\hat{B}$ и $\hat{C}[1]: \hat{B}[\boldsymbol{w}]=\operatorname{rot} \hat{P}[\boldsymbol{w}] ; \hat{C}[\boldsymbol{w}]=\left(p^{2} \varepsilon_{0} \mu_{0}-\operatorname{graddiv}\right) \hat{P}[\boldsymbol{w}]$.

Рассмотрим следующую систему интегро-дифференциальных уравнений [1]:

$$
\left\{\begin{array}{l}
\boldsymbol{u}+p \mu_{0} \hat{B}[(\mu(\boldsymbol{r})-1) \boldsymbol{v}]+\frac{1}{p \varepsilon_{0}} \hat{C}\left[\left(\sigma(\boldsymbol{r})+p \varepsilon_{0}(\varepsilon(\boldsymbol{r})-1)\right) \boldsymbol{u}\right]=\boldsymbol{U}_{0}, \\
\boldsymbol{v}-\hat{B}\left[\left(\sigma(\boldsymbol{r})+p \varepsilon_{0}(\varepsilon(\boldsymbol{r})-1)\right) \boldsymbol{u}\right]+\hat{C}[(\mu(\boldsymbol{r})-1) \boldsymbol{v}]=\boldsymbol{V}_{0}
\end{array}\right.
$$

где $\boldsymbol{U}_{0}=-\mu_{0} \hat{B}[\mu \boldsymbol{g}]-\frac{1}{p} \hat{C}[\varepsilon \boldsymbol{f}], \boldsymbol{V}_{0}=\varepsilon_{0} \hat{B}[\varepsilon \boldsymbol{f}]-\frac{1}{p} \hat{C}[\mu \boldsymbol{g}]$.

В силу свойств объемного потенциала [1] любое квадратично суммируемое в $\mathrm{R}^{3}$ решение (8) имеет квадратично суммируемый в $\mathrm{R}^{3}$ обобщенный ротор и удовлетворяет системе (6). Кроме того, систему (8) достаточно решить в пространстве $\left(\mathbf{L}_{2}(\Omega)\right)^{2}$ : в точках, внешних по отношению к $\Omega$, решение может будет получено из решения внутри $\Omega$ непосредственным интегрированием [1], причем в целом решение будет квадратично суммируемым в $\mathrm{R}^{3}$.

Докажем существование решения системы (8) в области $\Omega$. Рассмотрим следующую замену неизвестных функций:

$$
\left\{\begin{array}{l}
\boldsymbol{u}=\frac{p \sqrt{\varepsilon_{0}} \boldsymbol{U}}{\sigma(\boldsymbol{r})+p \varepsilon_{0}(\varepsilon(\boldsymbol{r})-1)}, \\
\boldsymbol{v}=\frac{\boldsymbol{V}}{\sqrt{\mu_{0}}(\mu(\boldsymbol{r})-1)} .
\end{array}\right.
$$

Заметим, что в силу свойств функций $\sigma(\boldsymbol{r}), \varepsilon(\boldsymbol{r})$ и $\mu(\boldsymbol{r})$, функции 
$\sigma(\boldsymbol{r})+p \varepsilon_{0}(\varepsilon(\boldsymbol{r})-1)$ и $\mu(\boldsymbol{r})-1$ непрерывны в $\Omega_{i}$ и $\Omega \backslash\left(\bigcup_{i=1}^{k} \bar{\Omega}_{i}\right)$, положительны и могут быть непрерывно продолжены с каждой из этих областей на ее границу, оставаясь при таком продолжении положительными. Следовательно, замена (9) осуществляет взаимно-однозначное отображение пространства $\left(\mathbf{L}_{2}(\Omega)\right)^{2}$ в себя.

С использованием (9) система (8) может быть приведена к виду:

$$
\left\{\begin{array}{l}
\frac{p \varepsilon_{0}}{\sigma(\boldsymbol{r})+p \varepsilon_{0}(\varepsilon(\boldsymbol{r})-1)} \boldsymbol{U}+p \sqrt{\varepsilon_{0} \mu_{0}} \hat{B}[\boldsymbol{V}]+\hat{C}[\boldsymbol{U}]=\sqrt{\varepsilon_{0}} \boldsymbol{U}_{0}, \\
\frac{1}{(\mu(\boldsymbol{r})-1)} \boldsymbol{V}-p \sqrt{\varepsilon_{0} \mu_{0}} \hat{B}[\boldsymbol{U}]+\hat{C}[\boldsymbol{V}]=\sqrt{\mu_{0}} \boldsymbol{V}_{0}
\end{array}\right.
$$

Исследуем левую часть полученной системы, учитывая самосопряженность оператора $\hat{B}$ и диссипативность (неотрицательную определенность) оператора $\hat{C}[1 ; 4]$. Умножим первое и второе уравнение системы (10) соответственно на $\boldsymbol{U}$ и $\boldsymbol{V}$; проинтегрируем по $\Omega$; затем сложим уравнения:

$$
\begin{gathered}
\left(\frac{p \varepsilon_{0}}{\sigma+p \varepsilon_{0}(\varepsilon-1)} \boldsymbol{U}, \boldsymbol{U}\right)+(\hat{C}[\boldsymbol{U}], \boldsymbol{U})+p \sqrt{\varepsilon_{0} \mu_{0}}(\hat{B}[\boldsymbol{V}], \boldsymbol{U})- \\
-p \sqrt{\varepsilon_{0} \mu_{0}}(\hat{B}[\boldsymbol{U}], \boldsymbol{V})+\left(\frac{1}{\mu-1} \boldsymbol{V}, \boldsymbol{V}\right)+(\hat{C}[\boldsymbol{V}], \boldsymbol{V}) \geq \\
\quad \geq\left(\frac{p \varepsilon_{0}}{\sigma+p \varepsilon_{0}(\varepsilon-1)} \boldsymbol{U}, \boldsymbol{U}\right)+\left(\frac{1}{\mu-1} \boldsymbol{V}, \boldsymbol{V}\right) \geq \\
\geq \frac{p \varepsilon_{0}}{\sigma_{\text {sup }}+p \varepsilon_{0}\left(\varepsilon_{\text {sup }}-1\right)} \cdot(\boldsymbol{U}, \boldsymbol{U})+\frac{1}{\mu_{\text {sup }}-1} \cdot(\boldsymbol{V}, \boldsymbol{V}) \geq \\
\geq \min \left\{\frac{p \varepsilon_{0}}{\sigma_{\text {sup }}+p \varepsilon_{0}\left(\varepsilon_{\text {sup }}-1\right)}, \frac{1}{\mu_{\text {sup }}-1}\right\} \cdot((\boldsymbol{U}, \boldsymbol{U})+(\boldsymbol{V}, \boldsymbol{V})),
\end{gathered}
$$

где круглыми скобками обозначено скалярное произведение в $\mathbf{L}_{2}(\Omega)$, определяемое через интеграл от произведения векторных функций.

Заметим, что дроби, полученные в (11), положительны; то есть положительна минимальная из них. Следовательно, в силу полученного оценочного неравенства у системы (10) существует единственное решение в $\left(\mathbf{L}_{2}(\Omega)\right)^{2}[1]$. Из этого вытекает, что у системы (6) существует решение в $\left(\mathbf{H}\left(\operatorname{rot}, \mathrm{R}^{3}\right)\right)^{2}$. Теорема доказана. 
С. В. Марвин. Начально-краевая задача электродинамики для дефектного ферритового тела

Из теорем 4 и 5 непосредственно вытекает, что начально-краевая задача (1)-(3) в выбранном функциональном классе имеет единственное решение, зависящее от начальных условий непрерывно по норме $\mathbf{L}_{2}\left(\mathrm{R}^{3}\right)$ [7].

\section{Заключение}

Полученные результаты, касающиеся существования и единственности решения задачи (1)-(3), имеют существенную прикладную значимость: установленные свойства оператора $\hat{A}$ полезны не только для доказательства существования и единственности решения, но также позволяют применять для поиска решения численные методы, аналогичные неявному методу Эйлера для обыкновенных дифференциальных уравнений [7]. В этом заключается основное направление дальнейших исследований по тематике данной работы.

\section{Литература}

1. Дякин В. В., Сандовский В. А. Задачи электродинамики в неразрушающем контроле. Екатеринбург: ИФМ УрО РАН, 2008. 390 с.

2. Дюво Г., Лионс Ж.-Л. Неравенства в механике и физике. М.: Наука, 1980. $384 \mathrm{c}$.

3. Калинин А. В. Математические задачи физической диагностики. Корректность задач электромагнитной теории в стационарном и квазистационарном приближении. Нижний Новгород: ГОУ ВПО ННГУ, 2007. 121 с.

4. Марвин С. В. Начально-краевая задача электромагнитного контроля дефектного ферромагнитного проводника остаточным полем мгновенно выключенного стороннего тока // Дефектоскопия. 2016. № 11. С. 27-38. DOI: 10.1134/S106183091611005X.

5. Марвин С. В. Начально-краевая задача для однородной системы уравнений Максвелла в случае магнитодиэлектрического тела с проводящими ферромагнитными включениями // Вестник Томского государственного университета. Математика и механика. 2017. № 47. C. 22-36. DOI: 10.17223/19988621/47/3.

6. Ильинский А. С., Кравцов В. В., Свешников А. Г. Математические модели электродинамики. М.: Высшая школа, 1991. 224 с.

7. Крейн С. Г. Линейные дифференциальные уравнения в банаховом пространстве. М.: Наука, 1967. 464 с.

\section{INITIAL BOUNDARY VALUE PROBLEM OF ELECTRODYNAMICS FOR A DEFECTIVE FERRITE BODY}

\section{Sergey V. Marvin}

Cand. Sci. (Phys. and Math.), Doctoral Candidate, A/Prof.,

The First President of Russia B. N. Yeltsin Ural Federal University

19 Mira St., Ekaterinburg 620002, Russia

The article considers initial boundary value problem for Maxwell's equations in relation to a ferrite body, which has some structural defects. For initial boundary value problem we have chosen a functional class that takes into account the match- 
ing conditions on the interface of two media, which are not perfect conductors. Vector fields of this functional class are square-integrable in the whole space and have square-integrable generalized rotors. In addition, vector fields are timedifferentiable in the sense of convergence in mean-square norm. Operating with broad assumptions about the dependence of electrical conductivity, dielectric and magnetic permeability of a ferrite on spaces coordinates, and natural assumptions about the dependence of external current on time, it has been shown, that in the given functional class there is one and only one solution of the considered initial boundary value problem, and that solution is continuous in the initial conditions.

Keywords: Maxwell's equations; initial conditions; matching conditions; meansquare norm; Banach space; existence theorem; generalized rotor; integrodifferential equations; closed operator; inverse operator.

\section{References}

1. Dyakin V. V., Sandovskii V. A. Zadachi elektrodinamiki v nerazrushayushchem kontrole [Problems of Electrodynamics in Nondestructive Testing]. Ekaterinburg: IMP UB RAS Publ., 2008. 390 p.

2. Duvant G., Lions J. L. Inequalities in Mechanics and Physics. Springer-Verlag Berlin Heidelberg, 1976. 384 p.

3. Kalinin A. V. Matematicheskie zadachi fizicheskoi diagnostiki. Korrektnost zadach elektromagnitnoi teorii $v$ statsionarnom $i$ kvazistatsionarnom priblizhenii [Mathematical Problems of Physical Diagnostics. The Correctness of Electromagnetic Theory Problems in Steady-State and Quasi-Steady-State Approximation]. Nizhnii Novgorod: Nizhny Novgorod State University Publ., 2007. 121 p.

4. Marvin S. V. Nachalno-kraevaya zadacha elektromagnitnogo kontrolya defektnogo ferromagnitnogo provodnika ostatochnym polem mgnovenno vyklyuchennogo storonnego toka [Initial Boundary Value Problem of Electromagnetic Control of a Defect Ferromagnetic Conductor by the Residual Field of an Instantaneous Switched-Off Extrinsic Current]. Russian Journal of Nondestructive Testing. 2016. No. 11. Pp. 27-38. DOI: 10.1134/S106183091611005X.

5. Marvin S. V. Nachalno-kraevaya zadacha dlya odnorodnoi sistemy uravnenii Maksvella $\mathrm{v}$ sluchae magnitodielektricheskogo tela s provodyashchimi ferromagnitnymi vklyucheniyami [Initial Boundary Value Problem for a Homogeneous System of Maxwell's Equations in the Case of a Magnetodielectric Body with Conducting Ferromagnetic Inclusions]. Vestnik Tomskogo gosudarstvennogo universiteta. Matematika $i$ mekhanika. 2017. No. 47. Pp. 22-36. DOI: 10.17223/19988621/47/3.

6. Ilinskii A. S., Kravtsov V. V., Sveshnikov A. G. Matematicheskie modeli elektrodinamiki [Mathematical Models of Electrodynamics]. Moscow: Vysshaya shkola, 1991. 224 p.

7. Krein S. G. Lineinye differentsialnye uravneniya $v$ banakhovom prostranstve [Linear Differential Equations in a Banach Space]. Moscow: Nauka Publ., 1967. 464 p. 